\title{
Corrigendum: Cost minimisation of product transhipment for physical distribution management
}

\author{
Authors: \\ Obioma R. Nwaogbe ${ }^{1}$ \\ Victor Omoke ${ }^{1}$ \\ Emmanuel C. Ubani ${ }^{2}$ \\ Sunday I. Ukaegbu ${ }^{3}$
}

\section{Affiliations:}

${ }^{1}$ Department of Transport Management Technology, Federal University of

Technology Minna, Nigeria

${ }^{2}$ Department of Project Management Technology,

Federal University of

Technology Owerri, Nigeria

${ }^{3}$ Department Transport

Management Technology,

Federal University of

Technology Owerri, Nigeria

Correspondence to:

Obioma Nwaogbe

Email:

obioma.nwaogbe@futminna.

edu.ng

Postal address:

Department of Transport

Management Technology,

Federal University of

Technology Minna, P.M.B 65

Minna, Niger State-Nigeria

Dates:

Published: 13 Feb. 2014

How to cite this article:

Nwaogbe, O.R., Omoke, V., Ubani, E.C. \& Ukaegbu, S.I., 2014, 'Corrigendum: Cost minimisation of product transhipment for physical distribution management', Journal of Transport and Supply Chain Management 7(1), Art. \#94, 1 page. http:// dx.doi.org/10.4102/jtscm. v7i1.94-1

Note:

Doi of original article: http:// dx.doi.org/10.4102/jtscm. v7i1.94
The original Table 2 on page 4 of 9 of the article contained errors. Please see below the correct table with the corrections given in bold:

TABLE 2: Quantity demanded by customers from depot or warehouse

\begin{tabular}{lccc}
\hline Customer or retailers & \multicolumn{2}{c}{ Quantity Demanded (in cartons per year) } & \multirow{2}{*}{ Total } \\
\cline { 2 - 3 } & Tura medicated & Tura supreme & 33739 \\
\hline Abia & 10935 & 22804 & $\mathbf{5 1 1 4}$ \\
Imo & $\mathbf{3 4 2 4}$ & $\mathbf{1 6 9 0}$ & $\mathbf{5 8 8 8}$ \\
Cross river & 560 & 5328 & $\mathbf{3 7 1 9}$ \\
Rivers & 2375 & 1344 & $\mathbf{1 8 4 0 5}$ \\
Onitsha & 6505 & 11900 & $\mathbf{1 2 8 6}$ \\
Ebonyi & 230 & 1056 & 1888 \\
Bayelsa & 360 & 1528 & $\mathbf{1 2 3 3 2}$ \\
Enugu & 934 & 11398 & $\mathbf{2 6 6 4}$ \\
Benue & 600 & 2064 & $\mathbf{1 0 3 1 5}$ \\
Akwa-lbom & 995 & 9320 & $\mathbf{7 0 1 6 6}$ \\
\hline Total & $\mathbf{2 5 1 8 4}$ & $\mathbf{9 5 3 5 0}$ \\
\hline
\end{tabular}

Source: E. Akogu (Godrej Nigeria Limited) pers. comm., 09 April 2012 